\title{
Formación del residente desde su abordaje como adulto
}

\author{
J.I. Ricarte-Díez, J.M. Martínez-Carretero (†)
}

La formación pregrado, posgrado y la formación continuada en medicina podemos encuadrarlas dentro de la educación para adultos. Si en la formación pregrado predomina la transmisión de conocimientos, la formación posgrado debería basarse en un asentamiento de esos conocimientos y en la adquisición de habilidades. Estas tareas, en el caso de la especialización médica vía MIR, se desarrollarán en un contexto concreto donde el residente se encontrará ante la necesidad de la resolución de problemas. Como adulto podrá responsabilizarse de su propia formación, pero necesita de la guía y orientación de un tutor, pieza clave en la educación y evaluación que no sólo mide los objetivos alcanzados, sino que permite mejorar las estrategias de aprendizaje. Tanto el tutor como los posibles profesionales sanitarios que participen en la formación son de vital importancia y merecen un reconocimiento, unos recursos y una formación acorde a su labor. En el presente artículo haremos un repaso del proceso formativo desde el pregrado hasta la residencia, y también destacaremos los aspectos evaluativos como indispensables en dicho proceso.

Palabras clave. Aprendizaje. Educación médica. Evaluación. Residencia.

\section{Resident's education from his adult's perspective}

Undergraduate, postgraduate and continuing medical education must be considered as adult education. If in undergraduate medical education dominates the transmission of knowledge, postgraduate education should be based on quantitative and qualitative increase of these knowledge and on acquisition of specific skills. These tasks, in medical specialization through residency programs will be developed in a concrete context where residents will face the need to solve real problems. As adult, he/she will be able to take responsibility on its own training but, at the same time, he/she will need guidance and orientation from the tutor. Tutorial methodology is a key piece in training and its evaluation; it doesn't only measures specific training achievements, but enhances the improving of individual learning strategies. Both tutor and other health professionals who play a role in residency program, are essential and deserve professional recognition, enough learning resources and specific teaching skills to cope with this responsibility. In this article, a revision is presented of the formative process from undergraduate to postgraduate education level in which evaluation as an unavoidable aspect in the above mentioned process is emphasized.

Key words. Assessment. Learning. Medical education. Residents training.

\section{La docencia en medicina}

Hasta el siglo xx, los textos de Hipócrates y las experiencias propias del ensayo-error seguían siendo la base de los conocimientos en medicina. Los posteriores cambios acontecidos se tradujeron también en la enseñanza de la medicina que debía adaptarse a los cambios vertiginosos y constantes que se sucedían en todos los campos del conocimiento [1]. El informe Flexner [2], que data de principios del siglo pasado, ya supuso un cambio interesante al aconsejar incorporar las especialidades en las facultades de medicina, aunque conllevó un distanciamiento de los médicos generales con su visión más integradora de los procesos patológicos. Así, se impuso un modelo centrado en la enfermedad, más hospitalario y teórico frente a la formación centrada en el paciente y orientada a la comunidad, en el modelo biopsicosocial o en la resolución de problemas [3]. En esta línea, los conocimientos científicos predominaban sobre el razonamiento clínico, la práctica, el desarrollo del carácter e incluso sobre actitudes como la compasión o la integridad [4] y se dejaba para el posgra-
Institut d'Estudis de la Salut (IES). Generalitat de Catalunya. Barcelona, España.

Correspondencia José I. Ricarte Díez. Institut d'Estudis de la Salut (IES). Generalitat de Catalunya. Roc Boronat, 81-95. E-08005 Barcelona.

Fax +34932386910

E-mail jiricarte@gencat.net 
duado el objetivo del desarrollo de competencias clínicas por encima de la transmisión de más conocimientos. La metodología de aprendizaje habitual solicitaba un importante esfuerzo de atención que ofrecía a los alumnos información cuantiosa cuya difícil memorización se evaluaría más tarde. Esta metodología era tomada por los alumnos como el medio que permitía superar la evaluación más que el hecho de aprender o mejorar. Prima el contenido sobre el proceso y se limita a una simple transmisión de información que se almacena como memoria sensorial. Lo que el alumno seleccione como más interesante pasará a la memoria a corto plazo, y si se vuelve a procesar puede quedar definitivamente en la memoria a largo plazo [5]. Así, con la memoria sensorial y una percepción de lo que es más interesante se puede retener mucho mejor la información. Todo ese proceso se invierte si con la práctica primero se comprende y después se memoriza. La formación no debería basarse en la memorización de datos sino en buscar una mayor relación con la práctica diaria, con lo cual se hace mayor hincapié en una formación desde la construcción del conocimiento. Las habilidades prácticas, por ejemplo, no se pueden adquirir sólo con el estudio, ya que requieren un entrenamiento con una duración proporcional a la dificultad de la habilidad que hay que entrenar [6]. Con esto no queremos despreciar el conocimiento pero sí afirmar que no es suficiente para la práctica médica si no se sabe integrar en cada paciente concreto y no se dispone de una serie de actitudes y habilidades clínicas, técnicas, interpersonales, además de unas buenas cualidades personales [7]. Con respecto a la vía MIR, se ha definido [8] como una formación para adquirir no sólo conocimientos sino también habilidades y valores necesarios para el pleno desarrollo de cada especialidad concreta a través de una práctica programada y supervisada. En esta fase de formación, el contenido, la metodología, las capacidades y las actitudes de alumno y profesor [9] son los factores que más van a determinar el proceso del aprendizaje. Estos factores en el mundo sanitario son distintos tanto por sus participantes (tutor y residente) como por el contexto asistencial concreto y propio. Los tutores deberían transmitir mediante la metodología adecuada tanto sus aptitudes (habilidades) como sus actitudes basadas en sus conocimientos teóricos y su experiencia práctica en el propio marco en el que trabajan. Se aprende más al usar los conocimientos y ponerlos en práctica que al recibirlos. La aparición de los pro- blemas en el contexto práctico hace que se recuerde e integre mucho mejor el conocimiento que si se adquiere por la exposición de un profesor en otro medio. Esto correspondería a lo que se denomina aprendizaje basado en problemas, que incentiva el desarrollo de una habilidad a partir de problemas o situaciones de salud similares a los que tendrán que enfrentarse en la realidad [10]. Es el propio residente el que decidirá qué necesita saber en función de tener que resolver los problemas que se le planteen, identificar la deficiencia surgida, buscar la solución y, por último, reflexionar sobre la aplicación práctica posible. La duda necesaria que debe reconocer lagunas propias no debe ser considerada como una falta de capacidad, sino que ha de ir seguida de una reflexión que permita generar un proceso en el que se revisen las competencias e implementen los posibles cambios necesarios en la formación [11]. Así, se puede hacer real el principio del learning by doing de William Halsted o el proverbio confuciano 'escucho y olvido, veo y recuerdo, hago y entiendo', en el que al mirar, ayudar y realizar procedimientos cada vez más complejos se adquieren las habilidades necesarias [12].

\section{La formación centrada en el que aprende}

Esta posibilidad de relación con lo que se quiere aprender en un contexto concreto permitirá establecer una valoración de la importancia y relevancia de los conocimientos que se aprenden y que a su vez determinarán el comportamiento en el mencionado contexto. Los estudiantes comienzan sin conocimientos médicos, de manera que emplean conceptos abstractos para éstos y reglas basadas en fórmulas. Según avanzan en la carrera, aplican esas fórmulas en situaciones concretas y reales. Ya en la residencia, la práctica madura el juicio clínico y dota de una visión integral a su labor. La experiencia que adquiere poco a poco incide, por lo tanto, en su respuesta a nuevos retos. Según el modelo analítico, el profesional, ante un paciente, genera una serie de sospechas diagnósticas que confirmará o descartará según la información que continúe adquiriendo basada en la anamnesis, la exploración o las pruebas complementarias (razonamiento clínico). La experiencia progresiva de los residentes se traduce en mejores hipótesis diagnósticas, de modo que además pueden explicar mejor el problema o usar 


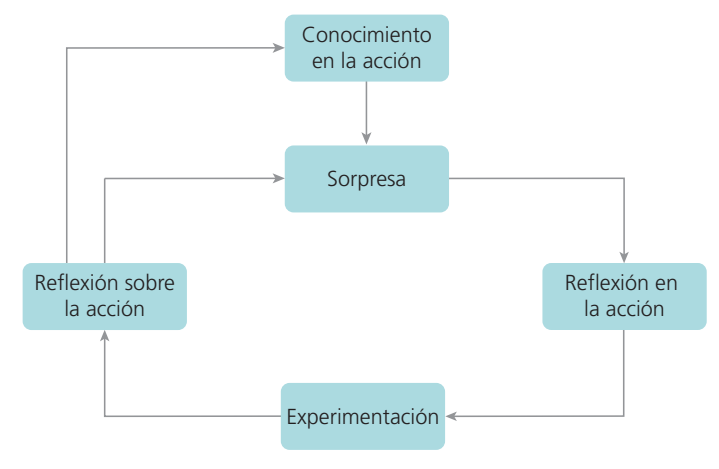

Figura. Aprendizaje según Schön (modificado de [15]).

la información y los datos de forma más adecuada [13]. Así, se llegó a plantear que, de alguna forma, la experiencia obtenida con la mayor exposición a los diversos casos clínicos influía en el razonamiento. Esto fue lo que se denominó reconocimiento de patrones o razonamiento no analítico. Supone comparar el nuevo problema con los ya resueltos anteriormente aunque no se garantice que responder bien un caso signifique la correcta solución de otros. Sin embargo, enfrentarse a un mayor número de casos clínicos aumentará la variabilidad y la experiencia para asumir otros similares. Entonces, la resolución del problema se basaría en un automatismo asentado en los conocimientos previos y el 'conocimiento en la acción' definido por Schön $[14,15]$, que correspondería con una especie de intuición. En esta dinámica no se pueden solucionar los nuevos casos más complejos, únicos e incluso contradictorios con los conocimientos adquiridos. Nuevamente se requiere de una reflexión que primero será 'sobre la marcha' (cuando el profesional descubre la situación) y después 'sobre la acción' (cuando se enfrenta a esa situación) (Figura). La reflexión que sucede con los nuevos casos ('sorpresa') y las experiencias previas son para Schön las que hacen aprender al individuo. Un especialista, con todo el tiempo trabajado y la experiencia acumulada, podrá hacer juicios clínicos rápidos de casos incluso atípicos [16]. La conciencia de nuestras propias virtudes como de los defectos favorecen esta actitud reflexiva y, a su vez, la reflexión nos hace darnos cuenta de esas virtudes y defectos. Con la rutina podríamos buscar más los puntos comunes de los casos nuevos con los ya conocidos y no iniciaríamos el proceso de la reflexión, de manera que perderíamos los beneficios del aprendizaje basado en problemas.

Por desconocimiento o por otros problemas ajenos, como la sobrecarga laboral, sería fácil volver a estrategias formativas o docentes que desaprovechen el contexto asistencial y que se basen sólo en una mera transmisión de información sobre la marcha sin haberla planificado ni preparado previamente $[17,18]$. No sirve el modelo por el que el residente aprende por el mero hecho de acompañar a su tutor y al cabo de 4 o 5 años, según la especialidad, ya esté capacitado. Son los propios tutores los que han denunciado [19] que no basta con el simple seguimiento de las actividades que pueden surgir en la actividad diaria sin ninguna planificación. La espontaneidad y la flexibilidad de la formación en la asistencia son importantes, ya que la adquisición de las competencias han de estar por encima del método o de cómo se llegue a esa formación con las rotaciones distintas requeridas, pero se han de concretar unos objetivos, contenidos y estrategias en el proceso de aprendizaje, de ahí la necesidad del feedback estructurado. Se puede integrar al residente en esa planificación como adulto que es. Si reconocemos la importancia de la responsabilidad del residente, debemos hacerle participar en el mayor número de tareas relacionadas con el aprendizaje. En el adulto podemos asumir un grado de responsabilidad que le permita asumir sus deberes, de tal manera que se implique de lleno en el proceso educativo. En este sentido, el profesor o tutor adquiere una posición horizontal y se convierte en un facilitador del conocimiento. Este papel activo del alumno rompe con el modelo paternalista o la posición vertical basado en la diferencia de conocimientos entre profesor y alumno [20]. La horizontalidad dota de un carácter de igualdad entre el profesor o facilitador y el alumno o participante. Se trata pues de una relación colaborativa y centrada en la que aprende que de forma progresiva va adquirir más responsabilidad. Así, el estudiante será el centro del proceso de aprendizaje. Construye, reconstruye, madura y organiza sus conocimientos previos sobre los que asentará nuevas experiencias. En nuestro caso, el residente, con toda una serie de conocimientos adquiridos durante la carrera, dispone de la oportunidad de asentar esos conocimientos y complementarlos con habilidades prácticas. La planificación o dirección en el adulto se basa en una maduración y un crecimiento ya 
Tabla. Métodos de enseñanza para la residencia.

\begin{tabular}{ll}
\hline \multirow{2}{*}{$\begin{array}{l}\text { Aprendizaje } \\
\text { de campo }\end{array}$} & Trabajo con los problemas en el contexto práctico observando al tutor o con la supervisión del tutor \\
\cline { 2 - 2 } & Asienta los conocimientos previos e incrementa éstos ante las experiencias con los problemas \\
\hline Autoaprendizaje & Estudio personal del propio residente \\
\cline { 2 - 2 } & Adquiere nuevos conocimientos o asienta los aprendidos en la práctica \\
\hline Trabajo en equipo & Interacción con otros residentes con los que puede intercambiar conocimientos \\
\hline Clases & Enseñanza unidireccional por una persona con conocimientos y experiencia \\
\hline Simulaciones & Transmisión de conocimientos y desarrollo de habilidades en un contexto práctico similar al real \\
\hline Talleres & Metodología práctica para la adquisición de habilidades en pequeños grupos
\end{tabular}

desarrollado, además de esta posibilidad de poder partir de unos conocimientos ya adquiridos que se han de enriquecer o perfeccionar. El papel activo recae en el que aprende y el docente es más facilitador que transmisor de conocimientos. Este proceso facilitador ha de suponer un continuo estímulo al alumno para que participe de forma activa, cubra sus necesidades y se comprometa en la tarea formativa por su interés e iniciativa [1]. El aprendizaje basado en problemas, que hemos comentado antes, será también un estímulo, ya que todo lo que sirva para la práctica diaria es considerado como útil y por lo tanto merecedor del esfuerzo necesario.

\section{Métodos de enseñanza}

El hecho de que la responsabilidad de la formación recaiga sobre el alumno [20] no significa que deba ser desestructurado o que deba ser abandonado a su suerte. La responsabilidad debe ir impulsada por una motivación personal interna [21] (que en el adulto es mayor que la motivación externa), pero es necesaria una estrategia orientadora (tutorización) que además ofrezca (facilite) la información de interés para el alumno con un ritmo adaptado a su capacidad y experiencia. El aprendizaje se realiza mediante la práctica supervisada y con la responsabilidad de un tutor con quien se comparte trabajo y pacientes. El desempeño de las actividades asistenciales junto con un incremento progresivo de su responsabilidad, que el propio residente va asumiendo, es la manera óptima de adquirir las competencias necesarias. En la misma situación podemos encontrar a otros residentes durante su proceso de formación. La interacción entre ellos es un elemento importante dentro del aprendizaje. Intervienen factores facilitadores de la formación como las elaboraciones, verbalizaciones, coconstrucción, soporte mutuo, crítica o una sintonía en el ámbito cognitivo y social [21]. En las metodologías activas entran en juego la participación y la reflexión de los propios alumnos como elemento esencial para avanzar en la formación. En coherencia con las teorías constructivistas [22], el conocimiento no se realiza en el 'interior' de las personas sino en el exterior, interaccionando con los otros y donde interviene un intercambio. Es decir, el aprendizaje viene de fuera al contrastar los conocimientos propios con los de los demás y así se incorporan nuevos puntos de vista. Con el conjunto de residentes es necesario establecer un clima de aprendizaje donde se sientan seguros, confortables y puedan expresarse. Para ello es útil, además del trabajo habitual con el tutor, las sesiones clínicas con casos reales o las sesiones bibliográficas.

A pesar de todo lo expuesto, nos encontramos con contextos muy variados, contenidos amplios, perfiles distintos, motivaciones, capacidades de 
los tutores (y residentes), variabilidad de recursos, etc. que pueden dificultar el objetivo del cumplimiento del programa y que habría que abordar de forma particular. Con el fin de facilitar el cumplimiento de los objetivos del programa de la residencia, recomendamos los siguientes métodos (Tabla):

- Aprendizaje durante el ejercicio profesional. El residente se encontrará frente a un problema que debe resolver en la situación real. Asentará sus conocimientos al tener que utilizarlos; además, deberá aprender a tomar decisiones y mejorar sus habilidades técnicas si se da el caso. Su grado de implicación puede ir desde la observación directa de lo que hace el tutor, la intervención tutorizada (con presencia del tutor) o la intervención directa (sin el tutor).

- Autoaprendizaje. El estudio sigue siendo necesario para la adquisición de nuevos conocimientos, así como para el mantenimiento de la competencia. Para ello también hace falta la implicación e iniciativa del residente, aunque puede ser estimulado mediante el encargo de tareas, la necesidad de resolución de problemas encontrados en la práctica diaria, la discusión de casos o la preparación de sesiones.

- Clases y simulaciones. El residente recibirá información unidireccional con el objetivo de aumentar los conocimientos sobre una materia concreta, tal como se hace en la formación pregrado. Puede ser útil para su práctica posterior en el contexto en el que está inmerso. En la metodología de la educación basada en problemas, se podrán utilizar simulaciones que se aproximen a la situación real.

- Trabajo en equipo. El residente aprovechará la existencia de otros iguales para profundizar en su aprendizaje mediante la interacción.

- Talleres. El objetivo no es tanto una transmisión de conocimientos sino la adquisición de habilidades en pequeños grupos.

\section{Influencia de la evaluación en el proceso formativo}

La evaluación es una parte inherente del proceso de aprendizaje. Es una necesidad tanto para el que aprende como para el que enseña. Sirve para comprobar el grado de adquisición de los cono- cimientos y habilidades, la consolidación de las competencias, el cumplimiento de los objetivos del programa, así como para detectar áreas de mejora conscientes o inconscientes [23]. Supone la parte final del proceso formativo o bien una intermedia si se quisiera modificar la enseñanza en función del resultado obtenido, ya que con la evaluación se pueden identificar problemas en el aprendizaje y promover una reflexión sobre ellos. No debe limitarse a evidenciar conocimientos sino al desempeño en situaciones concretas donde deban desarrollar sus capacidades. Si la evaluación se limita a los conocimientos de datos, la formación se orienta también hacia ese objetivo, ya que la forma de evaluar ejerce una influencia decisiva en el aprendizaje [24]. Se estudia con el pensamiento puesto en la forma de evaluar. Los cambios en su formato tienen un impacto innegable en la forma tanto de enseñar como de prepararse. Si consideramos que las destrezas son importantes y las evaluamos, podremos conseguir cambios en la metodología docente. Por su parte, los docentes también resultan evaluados junto a sus alumnos y pueden introducir mejoras en su labor. En resumen, la evaluación debe ser una herramienta que ayude a identificar lo que se ha aprendido y lo que queda por aprender [25], por lo que es un potente instrumento de mejora de la calidad de los programas de formación tanto en el proceso como en el resultado. Podemos distinguir dos métodos de evaluación: la evaluación formativa y la evaluación sumativa. La formativa se centra en el juicio de la suficiencia de lo que se ha pretendido enseñar en relación con un estándar mínimo previamente definido. El objetivo principal es generar círculos de mejora en la detección y resolución de problemas de aprendizaje, en los que tutor y residente aportarán pruebas de la consecución y aplicación del aprendizaje sobre la base de objetivos predefinidos y vinculados a competencias concretas [23]. Por su parte, la evaluación sumativa permite valorar la suficiencia de lo aprendido en relación con un estándar mínimo previamente definido. Pretende delimitar elementos de mejora docente que deberían introducirse tanto en los residentes como en la organización o el programa según los resultados obtenidos en la evaluación, y especialmente dirigido a asegurar la competencia. Los métodos son compartidos por los dos tipos de evaluación, aunque difiere la finalidad con la que se usan. La evaluación formativa tiene lugar 
durante todo el proceso de aprendizaje para mejorar el mismo, mientras que la sumativa se realiza al final de un aprendizaje para medir el nivel de formación alcanzado y acreditar la capacitación profesional. En nuestro medio no tenemos la costumbre de la evaluación formativa y se emplea más una evaluación sumativa basada en los conocimientos, que no mide la capacitación para la práctica, tiene un carácter punitivo, busca los datos excepcionales por encima de los básicos o prevalentes y no ofrece feedback constructivo ni facilita la formación continuada [7]. Surgen también voces que consideran que si el propio tutor realiza la evaluación sumativa puede perder la confianza de su residente. El Advisory Committee on Medical Training (ACMT), que regula en Europa la formación médica, emitió un informe en 1996 que explicita como 'es improbable que todas las habilidades y conocimientos requeridos de un especialista puedan ser calificados solamente mediante un examen (evaluación sumativa), por lo que es preferible que la valoración de la capacidad del residente se haga de manera continuada durante todo su período formativo (evaluación formativa)' [26]. Es una recomendación firme para que los residentes cuenten con un sistema de evaluación continuo que permita implementar mejoras. El programa docente de la Especialidad de Medicina Familiar y Comunitaria define la relación tutor-residente como un elemento clave en los resultados obtenidos de la formación posgraduada e incluso como el eje fundamental sobre el que gira todo el proceso formativo. Con estas intenciones, ha surgido la tutorización activa continua (TAC). Se trata de una metodología evaluativa del proceso formativo que se ha convertido en toda una estrategia de tutorización. Consiste en el desarrollo de entrevistas semiestructuradas donde se valoran los progresos, las deficiencias y los problemas organizativos [27]. El tutor participará durante todo el proceso formativo, de manera que podrá analizar la evolución del aprendizaje del residente e introducir medidas de mejora, y así superará la misión evaluativa anterior, de carácter más burocrático o administrativo, en la que se limitaba a un simple sumatorio de las calificaciones otorgadas al residente durante sus rotaciones. Requiere un esfuerzo específico del tutor porque según ésta puede tener que modificar tanto la planificación como el desarrollo de las actividades que el residente ha de realizar [28]. Se trata mucho más que de una mera recepción pasiva de los problemas o quejas de los residentes, ya que intenta mejorar la calidad de la docencia a lo largo de toda la residencia. Esta herramienta no tiene por objetivo calificar a los residentes y debe ser tomada por éstos como una ayuda, ya que corregirá errores que puedan cometer y además les estimulará a continuar con lo que ya hacen bien. En el nuevo programa de la especialidad de Medicina Familiar y Comunitaria ya aparece la recomendación de mantener 'un contacto estructurado y continuado con su tutor principal de atención primaria. La participación de un tutor no impide la existencia de otros profesionales sanitarios con quienes compartirá mucho tiempo y experiencias, y que también son básicos en la formación del residente. De ahí la importancia de la formación de los tutores en metodología docente dirigida fundamentalmente a la TAC y la valoración formativa. El papel del tutor resulta vital en el proceso de formación especializada, por lo que también es necesario acreditarlos y reconocer su tarea y responsabilidad.

Si hemos hablado de una metodología fundamentada en los 'problemas' para que se adquieran los conocimientos en un contexto real y práctico, también debemos hablar de una enseñanza centrada en 'el que aprende', que tendría en cuenta sus necesidades, deseos y expectativas. Este modelo se conoce como modelo ecológico y se opone a otro modelo centrado en el tutor. En este contexto de diálogo tutor-residente ha sido más fácil realizar la estrategia de valoración formativa que desde el año 1999 tiene lugar en las unidades docentes (UD) de Cataluña con la colaboración del Institut d'Estudis de la Salut (IES) del Departament de Salut de la Generalitat de Catalunya. Para ello se diseñaron dos instrumentos de valoración formativa [24]:

- Feedback entre tutor y residente. Se trata de entrevistas centradas en los intereses del que aprende. Analizan el conjunto de la actuación del residente en los distintos rotatorios y se negocian mejoras de aprendizaje. Se utilizan modelos de registro que transmitirán posibles propuestas a la unidad docente y plasmarán compromisos tanto del tutor como del residente.

- Sesiones de análisis del progreso competencial. Se realizan como mínimo cada seis meses y tienen una duración de 1 a 2 horas. Se utilizan pacientes simulados, maniquíes, casos por or- 
denador, búsquedas bibliográficas, self-audits o portafolios, entre otros métodos, en los cuales el residente y su tutor intentarán objetivar la adquisición de competencias previamente definidas.

En esta línea de evaluación formativa, en el año 2006 apareció el Libro del especialista en formación o libro del residente, en el que tiene un papel destacado el portafolio como instrumento, que recoge elementos que demuestran el aprendizaje y que, además, estimulan el aprendizaje basado en la autorreflexión.

\section{Conclusiones}

Hemos comenzado el presente artículo hablando del informe Flexner que surgió tras la preocupación por el incremento del número de facultades de Medicina en Estados Unidos, donde podían primar los intereses económicos frente a los sociales o formativos. Nuevamente, a pesar de la falta de profesionales en nuestro medio, la comunidad internacional ha expresado su preocupación por el desarrollo de facultades sin los suficientes recursos académicos o financieros que podrían debilitar la educación. Esta situación es extensible también a la formación de posgrado. Aparte de la labor asistencial indispensable que pueden realizar los residentes, una docencia de calidad también garantizará en el futuro unos profesionales de calidad, por lo que la docencia es uno de los pilares de cualquier sistema sanitario. La investigación y la docencia pueden marcar una diferencia importante si se toman en serio. Así, requerirían de una financiación y un reconocimiento parejos a su relevancia. A su vez, aunque la figura del tutor que debe organizar, orientar y evaluar el aprendizaje es imprescindible, no se han establecido unos criterios (ni recursos, ni formación) claros de quien debe asumir este rol y muchas veces ha recaído en quien ha mostrado una mejor predisposición. La formación especializada requiere, de alguna forma, que esta función se profesionalice y se dote de un reconocimiento, un tiempo y unos recursos, a la vez que se desarrollen instrumentos evaluativos de carácter formativo para detectar posibles deficiencias en el proceso de enseñanza.

\section{Bibliografía}

1. Granero-Molina J, Pérez-Ruiz IM, Muñoz-París MJ, Alcalde-Góngora E, Fernández-Sola C, Heredia-Berciano M. Propuesta docente para el aprendizaje del proceso de lavado quirúrgico. Enferm Clin 2003; 13: 53-8.

2. Flexner A. Medical education in the United States and Canada: a report to the Carnegie Foundation for the advancement of teaching. Boston: Updike; 1910.

3. Bonal P, Casado V. La medicina de familia como disciplina académica y la atención primaria como entorno de aprendizaje. Aten Primaria 2004; 34: 433-6.

4. Irby DM, Sullivan W, Ludmerer KM. American medical education 100 years after the Flexner report. N Engl J Med 2006; 355: 1339-44.

5. Good T, Brophy P. Psicología educativa contemporánea. México DF: McGraw-Hill Interamericana; 1996.

6. Fernández-Lobato R, Tartas-Ruiz A, Jiménez-Miramón FJ, Fradejas JM, Marín JM, Ruiz de Adana JC, et al. Curva de aprendizaje en la técnica de Stoppa. Cir Esp 2002; 72: 152-6.

7. Martínez-Altarriba MC. Evaluación de la competencia. Qué es y por qué realizarla. Semergen 2003; 29: 591-8.

8. Nebot-Cegarra J, Campillo M, Pérez J. Influencia de la metodología docente en la adquisición rápida de conocimientos. Educ Med 2003; 6: 37-43.

9. Sabater-Ortí L. Hacia un nuevo modelo de tutor de residentes. Cir Esp 2006; 80: 121-2.

10. Echevarría-Ruiz de Vargar C, Pérez-Castilla J, González López-Arza MV. La enseñanza de la medicina basada en la evidencia. Rehabilitación 2001; 35: 397-401.

11. Nolla-Domenjó M. Formación continuada: el proceso cognitivo y el aprendizaje profesional. Educ Med 2006; 9: 11-6.

12. Folse JR. Surgical education -adressing the challenges of change. Surgery 1996; 120: 575-9.

13. Norman G. Research in clinical reasoning: past history and current trends. Med Educ 2005; 39: 418-27.

14. Coles C. Approaching professional development. J Contin Educ Health Prof 1996; 16: 152-8.

15. Schön D. La formación de los profesionales reflexivos. Barcelona: Paidós; 1992.

16. Epstein RM. Assessment in medical education. N Engl J Med 2007; 356: 387-96.

17. Hekelman FP, Flynn, Glaver P, Galaska S, Philips JD. Peer coaching in clinical teaching: formative assessment of a case. Eval Health Prof 1994; 17: 366-81.

18. Irby DM. Teaching and learning in ambulatory care settings: a review of the literature. Acad Med 1995; 70: 898-931.

19. Loayssa-Lara JR, Monreal-Hijar A. La guía educativa del área clínica: un instrumento para una tutoría más sistemática y activa. Aten Primaria 2003; 31: 407-8.

20. Davies P. Approaches to evidence-based teaching. Med Teach 2000; 22: 14-21. 
21. Dolmans D, De Grave W, Wolfhagen I, Van der Vleuten C. Problem-based learning: future challenges for educational practice and research. Med Educ 2005; 39: 732-41.

22. Burr V. An introduction to social constructionism. London: Routledge; 1999.

23. Torán-Monserrat $\mathrm{P}$, Arnau-Figueras J. El porfolio como instrumento de valoración del residente. Aten Primaria 2006; 37: 371-3.

24. Ezquerra M, Bundo M, Forés-García D, Cots-Yago JM, Casada-Plana A, Fornells-Vallés JM. Desarrollo de un sistema de valoración formativa en las Unidades de Medicina Familiar y Comunitaria de Cataluña. Tribuna Docente 2004; 5: 16-23.
25. Prados-Castillejo JA. Preguntas sobre la evaluación de las competencias y alguna respuesta: portfolio. Aten Primaria 2005 ; 36: 3-4.

26. Tutosaus JD. Sistemas de evaluación de los residentes: rol del tutor en los mismos. Educ Med 2003; 6: 8-10.

27. Martín-Zurro A, Bundo M, Buxadé-Martí I, Casasa-Plana A, Cots-Yago JM, Ezquerra-Lezcano M, et al. Guia per a la tutorització activa continua (TAC). Unitats Docents de MFiC de Catalunya; enero 2004.

28. Sant-Arderiu E, Casajuana-Brunet J, Pou-Vila R, Aragonés-Forés R, Benítez-Camps $\mathrm{M}$. Tutorización activa continua: una metodología para intentar ser tutor durante los 3 años de la especialidad. Aten Primaria 2005; 36: 214-20. 